\title{
Industry Applications of Computational Intelligence
}

\author{
Runliang Dou ${ }^{1,2}$, Chen-Fu Chien ${ }^{3}$, Imed Kacem ${ }^{4}$, and Chia-Yu Hsu ${ }^{5 *}$ \\ ${ }^{1}$ Department of Information Management and Management Science, Tianjin University, China \\ E-mail:drl@tju.edu.cn \\ ${ }^{2}$ TEDA Jingnuo Hanhai Data Tech. Co. LTD, China \\ ${ }^{3}$ Department of Industrial Engineering and Engineering Management, National Tsing Hua University, Taiwan \\ E-mail: cfchien@mx.nthu.edu.tw \\ ${ }^{4}$ Université de Lorraine - LCOMS, France \\ E-mail: imed.kacem@univ-lorraine.fr \\ ${ }^{5}$ Department of Information Mangement, Yuan Ze University, Taiwan \\ E-Mail:cyhsu@saturn.yzu.edu.tw
}

\section{Preface}

Most companies in the manufacturing and service industries are faced with the challenge of making effective decisions in the context of big data. Big data has been accumulating due to fully automated manufacturing facilities, logistics and planning systems as well as service systems for business integration. Various techniques in computational intelligence and data science have been developed to extract useful information from the growing complexity, amount, and heterogeneity of these data.

This special issue considers the recent developments and empirical studies of industry applications of computational intelligence. In particular, the interest lies in examining the research issues concerned with quantitative and other modelling techniques to improve the decision quality and management effectiveness for manufacturing and service industries. Original, high quality papers on the theoretical developments and empirical studies with scientific novelty and insights were invited from scholars in various fields for review and publication. We sought papers that present new research contributions in the fields, such as production scheduling, equipment monitoring and fault diagnosis, medical and healthcare, and productivity evaluation, preferably illustrated with real world examples. All the submitted manuscripts were carefully blind peer reviewed by at least two referees, in accordance with this journal's review procedure, and finally, five papers were selected for this special issue.

The first paper, "Reduction of carbon emissions and total late work criterion in job shop scheduling by applying a multi-objective imperialist competitive algorithm" by Hamed Piroozfard, Kuan Yew Wong and Manoj Kumar Tiwari introduces a multi-objective imperialist competitive algorithm (MOICA) imitating the behavior of imperialistic competition for a low-carbon based multi-objective job shop scheduling problem. The numerical results reveal that the MOICA outperforms multi-objective particle swarm optimization (MOPSO) and the non-dominated sorting genetic algorithm II (NSGAII) in terms of quality metric, mean ideal distance, diversification metric, spacing metric, and number of non-dominated solutions.

The second paper, "A new approach for condition monitoring and detection of rail components and rail track in railway" by Mehmet Karakose, Orhan Yaman, Kagan Murat and Erhan Akin proposes a framework with image processing methods for the detection of railway components and monitoring of rail line structure. An empirical study with a test vehicle on a railway line was conducted and the results reveal that the possible faults can be prevented via early detection.

* Corresponding author. 
The third paper, "Dynamic deep learning algorithm based on incremental compensation for fault diagnosis model" by Jing Liu, Yacheng An, Runliang Dou and Haipeng Ji deals with fault diagnosis of equipment through the application of a dynamic deep learning algorithm based on an incremental compensation (ICDDL) model. Additionally, the support vector machine (SVM) algorithm is employed to classify the weighted models in a supervised way, and the backpropagation (BP) algorithm is utilized to fine tune the model. To evaluate the performance of the proposed method, the fault diagnosis for bearing equipment was used. The results show that the proposed ICDDL model has higher accuracy and lower computation time when compared to the denoising autoencoder (DAE), autoencoder, SVM and BP methods.

The fourth paper, "A novel two-step feature selection based cost sensitive myocardial infarction prediction model" by Atefeh Daraeil and Hojjatollah Hamidi proposes a cost sensitive sequential minimal optimization (SMO) model for Myocardial Infarction prediction. Moreover, a two-phase feature selection procedure was applied for solving class imbalance in Myocardial Infarction. The results of a case study conducted by heart specialists in an Iranian hospital, reveal that the proposed SMO model with the feature selection process has high prediction accuracy and sensitivity.

The fifth paper, "An agile mortality prediction model: hybrid logarithm least-squares support vector regression with cautious random particle swarm optimization" by Chien-Lung Chan, Chia-Li Chen, Hsien-Wei Ting and Dinh-Van Phan integrates logarithm least-squares support vector regression (LLS-SVR) and cautious random particle swarm optimization (CRPSO) for mortality prediction. The proposed LLS-SVR-CRPSO can rapidly provide critical support information for physicians' intensive care decision-making.

This special issue successfully addresses some critical research needs regarding industry applications of computational intelligence for emerging research needs for future production and services. We would like to express our sincere appreciations to all of the authors, the anonymous reviewers, and the Editors of the International Journal of Computational Intelligence Systems, for their invaluable contributions and great support in the production of this special issue. 